\title{
INHIBITION OF ADVANCED GLYCATION END-PRODUCT FORMATION BY QUERCETIN AND CATECHIN: AN ALTERNATIVE THERAPY FOR TREATING DIABETIC COMPLICATIONS
}

\author{
ANGELINE JULIUS, WAHEETA HOPPER* \\ Department of Biotechnology, School of Bioengineering, Faculty of Engineering and Technology, SRM University, Chennai, Tamil Nadu, \\ India. Email: waheeta.h@ktr.srmuniv.ac.in
}

Received: 26 April 2017, Revised and Accepted: 18 July 2017

\section{ABSTRACT}

Objective: The objective of this research was to determine early advanced glycation end-product (AGE) inhibition by natural aldose reductase inhibitors (ARIs), quercetin and catechin.

Methods: The assay mixture ( $4 \mathrm{ml}$ ) consisted of $2 \mathrm{ml}$ of $50 \mathrm{mM}$ phosphate-buffered saline (pH 7.4), $50 \mu \mathrm{g} / \mu \mathrm{lbovine} \mathrm{serum} \mathrm{albumin} \mathrm{(BSA),} \mathrm{and} 2 \mathrm{mM}$ glucose with or without the inhibitor. The test samples were treated with three different concentrations (10 mM, $20 \mathrm{mM}$, and $40 \mathrm{mM})$ of quercetin and catechin. High-throughput screening-based assay was adapted to perform the BSA-glucose test to determine the induction of AGE formation and its inhibition by quercetin, and catechin, using the fluorescence of the AGE-BSA sample at excitation and emission wavelengths of 350 and 450 nm.

Result: The ARIs, quercetin and catechin inhibited early glycation with an inhibitory concentration value of $15.58 \mathrm{mM}$ and $35.01 \mathrm{mM}$, respectively.

Conclusion: The suppression of AGEs formation by natural inhibitors of aldose reductase would provide an alternative approach to the control of diabetic complications.

Keywords: Aldose reductase inhibitors, Advanced glycation end-products, High-throughput virtual screening, Natural compounds, Diabetic complications.

(C) 2017 The Authors. Published by Innovare Academic Sciences Pvt Ltd. This is an open access article under the CC BY license (http://creativecommons. org/licenses/by/4. 0/) DOI: http://dx.doi.org/10.22159/ajpcr.2017.v10i11.19412.

\section{INTRODUCTION}

Diabetes is a life-threatening metabolic disorder, causing 1.5 million deaths per year worldwide [1]. One of the two patients with diabetes is diagnosed with complications, ranging from one to several [2], caused due to chronic hyperglycemia and inadequate treatment. The non-enzymatic glycation between the sugar and the protein is related to the development of diabetic complications. Advanced glycation end-products (AGEs), the products of glycation, are generated in response to the stimulations from different triggering mechanisms. The receptor of AGE (RAGE), being a signal transduction receptor, can activate various signal transduction cascades. AGE-RAGE interaction modulates the expression of various proinflammatory molecules. AGE-RAGE interaction activates nuclear factor-kappa B (NF- $\kappa \mathrm{B})$ and the key target of reactive oxygen species (ROS) generation in the cell. Furthermore, the upregulation of NF-KB further stimulates RAGE generation. Apart from RAGE activation, ROS generation and generation of AGE cause activation of the polyol pathway by the induction of aldose reductase (ALR2) and the rate-limiting enzyme of the pathway. The 3-deoxyglucosone produced as a by-product from the polyol pathway is a major precursor of AGE. Hyperglycemia-induced activation of ALR2 increases AGE production that in turn stimulate further activation of ALR2 [3].

The activation of polyol pathway, increased production of AGEs, NF$\kappa \mathrm{B}$ activation, activation of protein kinase $\mathrm{C}$, and oxidative stress are the main causes for the hyperglycemia induced vascular damage and the occurrence of diabetic complications. Although many factors could be responsible for the pathogenesis of diabetic complications, accumulation of AGEs plays the main role in inflammation, diabetes, and aging [4].

Glucose is a potent glycation agent and fructose, the by-product of the polyol pathway, causes severe glycation than glucose. AGE is increasingly found in the extracellular matrix, and the increased production of intracellular AGE precursors cause devastating effects in the body. Initially, a Schiff base is formed by the non-enzymatic reaction between the aldehyde group of sugar and the amino group of the protein. The rearrangement of Schiff base produces Amadori products that undergo rearrangements to form stable AGEs. This reaction is called Maillard reaction [5]. The formation of dicarbonyls such as 3-deoxyglucosone, methylglyoxal (MGO) and glyoxal occur in early glycation. The generation of the dicarbonyls, the advanced glycation precursors, occurs as early as 3 weeks due to slow degradation of glucose at $37^{\circ} \mathrm{C}$. Short increase in glucose level also provoked an increase in the generation of advanced glycation precursors [6]. In prolonged hyperglycemia, increased amounts of AGEs are found in the extracellular matrix causing a severe impact on the cellular function [7]. Damage is mainly due to the modification and mutation of intracellular proteins and the binding of modified plasma proteins to the RAGE, a signal transduction receptor found on the vascular endothelial cells and smooth muscle cells. Modification of intracellular proteins and plasma proteins damage the cell by the induction of ROS which in turn activates the NF- $\mathrm{KB}$ and upregulates the inflammatory pathways, causing several pathological changes [8].

The Maillard reaction is the resultant of the nonenzymatic reaction occurring between the proteins and the reducing sugars. A reducing sugar like glucose reacts with the amines to form unstable, Schiff base that rearranges to form stable amadori product. Dicarbonyl compounds, MGO, and deoxyglucosone are the resultant of the chemical transformation of the amadori products. During the last stages of glycation, AGEs are formed due to the oxidation and dehydration reactions which could cause several diabetic complications. AGEs are associated with diseases such as diabetes, renal failure, and cancers. The accumulation of AGEs on the proteins impairs the protein function and activity by mutating it. Increased AGE concentration in the tissue and the plasma compromises the antioxidant defense system causing 
deleterious effects in the body [9]. Since the discovery of Maillard reaction and the deleterious damage, it causes to the body, progress in identifying inhibitors that could inhibit nonenzymatic browning of proteins are of great interest [10].

The bovine serum albumin (BSA)-glucose test is a prominent method for detecting the amount of AGEs generated, using its fluorescence at excitation and emission wavelengths of 350 and $450 \mathrm{~nm}$ [11]. Induction of AGEs and its inhibition by the plant extracts or the pure compound could be used effectively to determine the AGE inhibition property of the compounds.

Traditional medicine has used different sources of food that act as natural free radical scavengers to cure various diseases. Several natural and synthetic inhibitors of ALR2 and AGEs show effective prevention of diabetic complications in vivo with experimental models and clinical trials [12]. Quercetin, luteolin, and kaempferol, the flavonoids from the whole plant extract of Saraca indica, proved to have rat lens ALR2 inhibiting activity. The structure-activity relationship of the flavonoids inhibiting both ALR2 and AGEs has been studied to identify natural compounds that could potentially inhibit both the factors.

Treatment using natural medicines is preferred as an alternative source for treating aging and diabetes. Herbal medicines are used extensively for preventing and treating diseases since they possess antidiabetic, antihyperlipidemic, and anti-inflammatory properties [13,14]. Early glycation can be prevented by preventing the formation of precursors of AGEs. Polyphenols such as genistein, naringin, and ellagic acid are reported to control aldehyde-induced glycation and prevention of the formation of AGE precursors [15]. This work adapts a cost-effective high-throughput screening-based assay to identify the potency of aldose reductase inhibitors (ARIs), quercetin, and catechin in the prevention of early AGEs [16].

\section{METHODS}

All the chemicals from were purchased from Sigma-Aldrich.

\section{AGE inhibition assay}

The measurement of AGE formation and its inhibition by test compounds were performed according to Matsuura et al. [17]. The inhibition of the Maillard reaction by the test samples was determined by the decrease in fluorescence in comparison with the positive control without the drug treatment. The pure compounds, quercetin and catechin, were dissolved in dimethyl sulfoxide. The assay mixture $(4 \mathrm{ml})$ consisted of $2 \mathrm{ml}$ of $50 \mathrm{mM}$ phosphate-buffered saline (PBS) ( $\mathrm{pH} 7.4$ ), $50 \mu \mathrm{g} / \mu \mathrm{l} \mathrm{BSA}$, and $2 \mathrm{mM}$ glucose with or without the inhibitor. The negative control was prepared without the glucose and the inhibitor. Positive control did not contain the inhibitor. The test samples were treated with three different concentrations $(10 \mathrm{mM}, 20 \mathrm{mM}$, and $40 \mathrm{mM})$ of quercetin and catechin. The negative control (blank) was kept at $4^{\circ} \mathrm{C}$ until measurement. The assay mixtures were incubated for $24 \mathrm{hrs}$ in the dark. $500 \mu \mathrm{l}$ of the aliquots were transformed to $1.5 \mathrm{ml}$ Eppendorf Tube, and $50 \mu \mathrm{l}$ of $100 \%$ trichloroacetic acid was added. The contents were vortexed and centrifuged at $15000 \mathrm{rpm}$ at $4^{\circ} \mathrm{C}$ for 4 minutes. The supernatant was discarded, and the AGE-BSA precipitate was dissolved in $1 \mathrm{ml}$ of PBS, and the samples were screened using spectrofluorometer to determine AGE formation in the sample. The samples were loaded in triplicates to a 96-well black plate. Changes in the intensity of the fluorescence detected in the test samples were compared with the fluorescence of the untreated control. The fluorescence end point was read at excitation, 360 and emission, 460 wavelengths and was directly proportional to the AGEs in the sample.

The inhibition percentage of AGE was calculated by the formula:

Percentage of inhibition $=\frac{(\text { Positivecontrol }- \text { test })}{\text { Positive control }} \times 100$

\section{RESULTS}

\section{AGE inhibition assay}

AGE inhibition was determined for the two known natural ARIs, quercetin and catechin. Table 1 lists the AGE inhibition assay reaction contents and quantities. Inhibitory concentration $\left(\mathrm{IC}_{50}\right)$ was calculated by plotting the concentration of the compound against its inhibition, using linear regression graph. $\mathrm{IC}_{50}$ was calculated using the slope of the graph. Better fit of the regression line was observed with regression values $\left(R^{2}\right)$ of 0.96 and 1 for quercetin and catechin, respectively. The experiment was repeated thrice for concordancy. IC $_{50}$ values of quercetin and catechin were $15.58 \mathrm{mM}$ and $35.01 \mathrm{mM}$, respectively. Fig. 1 illustrates the percent inhibition of AGE by quercetin and catechin at three different concentrations.

\section{DISCUSSION}

Hyperglycemia induces the formation of AGEs through various pathways, including the polyol pathway. The activation of ALR2 and the first rate-limiting enzyme of the polyol pathway have been reported to be involved in increased generation of AGEs. Diet, rich in protein and fat, is the external source of AGEs that is proportional to the increased serum levels of AGE which contribute to the crosslinking of the proteins, worsening the complications in patients with diabetes [18]. Increased expression of mitogen-activated protein kinases, NF- $\mathrm{kB}$, and vascular cell adhesion molecule have occurred in patients with AGE-rich diet, indicating the probability of proliferative diseases [19]. Crude plant extracts reported having AGE inhibition activity have been successful in the control and management of diabetes and its complications along with regulating blood glucose [20]. Although most of the synthetic inhibitors of AGEs were withdrawn due to side effects, phenolic compounds present in plants which are identified to have potential AGE inhibiting activity [21,22]. The whole plant extracts of Ligularia fischeri were reported to have ALR2, AGEs inhibition, and radical scavenging activity, contributed by the compound 3, 4-dicaffeoylquinic acid [23]. Glycyrrhiza glabra, Withania somnifera, Petroselinum crispum, and Terminalia chebula have shown benefecial effects in the treatment of diabetes and complications by inhibiting the formation of AGEs [24]. Eugenol, a phenylpropanoid from Ocimum Gratissimum, is an FDAapproved inhibitor of AGE that can be used for the management of diabetes and complications [25]. Compounds with AGE inhibiting property also act as natural ROS scavengers $[26,27]$. Polyol pathwayinduced AGE formation might be controlled by inhibiting the activity of ALR2. Natural ALR2 inhibitors with AGE suppressing potency and antioxidant properties would be a three-sided useful approach to control diabetic complications. Dietary flavonoids are used to suppress or control the occurrence of chronic diseases such as cancers and diabetes by preventing ROS formation and by supplying antioxidants that would

Table 1: Reaction contents of AGE inhibition assay

\begin{tabular}{|c|c|c|c|c|c|c|c|c|}
\hline Reagents & Positive control & Negative control & Q $10 \mathrm{mM}$ & Q $20 \mathrm{mM}$ & Q $40 \mathrm{mM}$ & C $10 \mathrm{mM}$ & C $20 \mathrm{mM}$ & C $40 \mathrm{mM}$ \\
\hline PBS (ml) & 2 & 2 & 2 & 2 & 2 & 2 & 2 & 2 \\
\hline $\mathrm{BSA}(50 \mathrm{mg} / \mathrm{ml})$ & 800 & 800 & 800 & 800 & 800 & 800 & 800 & 800 \\
\hline 2 mM Glucose $(\mu \mathrm{l})$ & 400 & 0 & 400 & 400 & 400 & 400 & 400 & 400 \\
\hline Inhibitor $(\mu \mathrm{l})$ & 0 & 0 & 800 & 800 & 800 & 800 & 800 & 800 \\
\hline Distilled water $(\mu \mathrm{l})$ & 800 & 1200 & 0 & 0 & 0 & 0 & 0 & 0 \\
\hline
\end{tabular}

Q: Quercetin, C: Catechin, AGEs: Advanced glycation end-products, BSA: Bovine serum albumin, PBS: Phosphate-buffered saline 


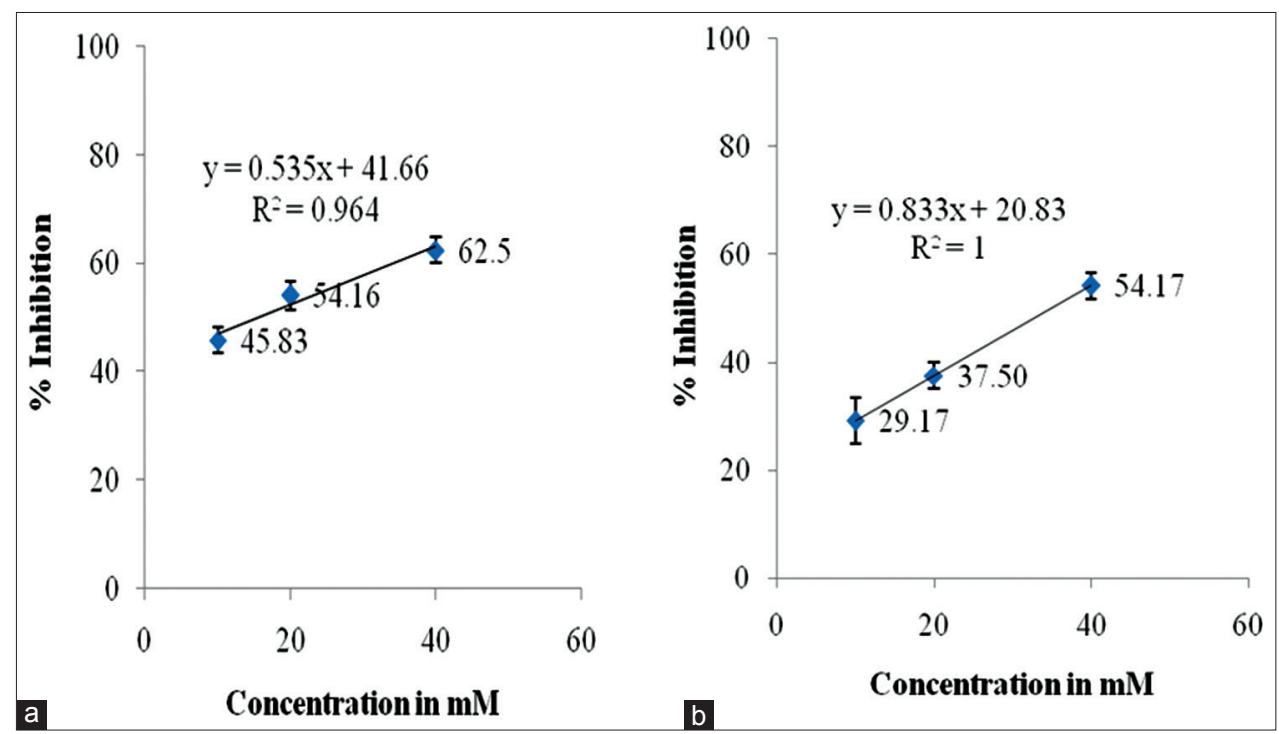

Fig. 1: Inhibitory concentration (IC ${ }_{50}$ ) prediction of (a) quercetin and (b) catechin using linear regression graph plotted with \% inhibition data. The $\mathrm{IC}_{50}$ values are expressed as mean \pm standard deviation for $\mathrm{n}=3$

quench the ROS. Increasing the antioxidant defense system would suppress the damage by the ROS in the human body. The flavonoids, quercetin, and catechin had antioxidant property, antiproliferative activity, and antioxidant properties [28] when tested on cancer cell lines of colon (Caco-2) breast (MCF-7), and pancreas (BxPC-3) [29]. The potency of quercetin and catechin to inhibit ALR2 [30,31] is already well known, and this study reveals their potential in suppressing the formation of AGEs in a $24 \mathrm{hrs}$ early glycation assay involving BSA and glucose.

Accumulation of AGE during hyperglycemia and its degradation with proper glycemic control indicate the induction of AGE during hyperglycemia. AGE-RAGE formation, further, accelerates the formation of AGE, during hyperglycemia [32]. Furthermore, AGE-RAGE-induced ROS generation causes worsening of vascular complications in diabetic patients. Progression of diabetic nephropathy has been controlled by preventing ROS production induced by cytosolic AGE-RAGE, inhibiting mitochondrial superoxide production in hyperglycemic conditions [33]. Therefore, the importance of the suppression or control of AGE formation during hyperglycemia is vital to prevent worsening of the disease. In this study, quercetin and catechin, the known natural inhibitors of ALR2, were assayed for AGE inhibition, induced using incubation of BSA with glucose. The fluorescence was proportional to the formation of yellow AGE-BSA precipitate that decreased with the treatment of quercetin and catechin over incubation. Although various attempts have been made to identify effective glycation inhibitors, and few natural compounds have been proven to suppress AGE production, after the discovery of aminoguanidine that had the capacity to prevent the diabetes-induced formation of AGEs. The suppression of AGEs formation by natural inhibitors of ALR2 such as quercetin and catechin would provide an alternative approach to the control of diabetic complications.

\section{CONCLUSION}

Diabetic complications have been a major problem more than diabetes itself since it affects both small and large blood vessels, causing deleterious effects. The pathology of diabetic complications is caused due to multiple factors, among which activation of polyol pathway, generation of AGEs and ROS plays a vital role. ALR2 inhibiting flavonoids, quercetin and catechin inhibit AGE formation at $\mathrm{IC}_{50}$ concentrations of $15.58 \mathrm{mM}$ and $35.01 \mathrm{mM}$, respectively. This indicates the potency of quercetin and catechin as AGE inhibitors, apart from ALR2 inhibition, which could be used to suppress diabetes-induced AGE formation and to control worsening of diabetic complications.

\section{ACKNOWLEDGMENTS}

The authors thank SRM University for providing facilities and financial support.

\section{REFERENCES}

1. Available from: http://www.who.int/mediacentre/factsheets/fs355/en.

2. Forssas E, Arffman M, Manderbacka K, Keskimäki I, Ruuth I, Sund R. Multiple complications among people with diabetes from Finland: An 18-year follow-up in 1994-2011. BMJ Open Diabetes Res Care 2016;4(1):e000254.

3. Giacco F, Brownlee M. Oxidative stress and diabetic complications. Circ Res 2010;107(9):1058-70.

4. Yan SF, Ramasamy R, Schmidt AM. The receptor for advanced glycation endproducts (RAGE) and cardiovascular disease. Expert Rev Mol Med 2009;11:e9.

5. Sadowska-Bartosz I, Bartosz G. Prevention of protein glycation by natural compounds. Molecules 2015;20(2):3309-34.

6. Thornalley PJ, Langborg A, Minhas HS. Formation of glyoxal, methylglyoxal and 3-deoxyglucosone in the glycation of proteins by glucose. Biochem J 1999;344:109-16.

7. Stitt AW, Moore JE, Sharkey JA, Murphy G, Simpson DA, Bucala R, et al. Advanced glycation end products in vitreous: Structural and functional implications for diabetic vitreopathy. Invest Ophthalmol Vis Sci 1998;39(13):2517-23.

8. Tangvarasittichai S, Deebukkhum S, Tangvarasittichai O. Progression of increased oxidative stress and inflammation in chronic kidney disease patients with Type 2 diabetes mellitus. Int J Pharm Clin Res 2016;8(6):596-603.

9. Lee HS, Hong CO, Lee KW. Advanced glycation endproduct-induced diabetic complications. Food Sci Biotechnol 2008;17(6):1131-8.

10. More TA, Kulkarni BR, Nalawade ML, Arvindekar AU. Antidiabetic activity of linalool and limonene in streptozotocin-induced diabetic rat: A combinatorial therapy approach. Int J Pharm Pharm Sci 2014:6(8):159-63.

11. Adeshara KA, Diwan AG, Tupe RS. Diabetes and complications: Cellular signaling pathways, current understanding and targeted therapies. Curr Drug Targets 2016;17(11):1309-28.

12. Younus H, Anwar S. Prevention of non-enzymatic glycosylation (glycation): Implication in the treatment of diabetic complication. Int $\mathrm{J}$ Health Sci (Qassim) 2016;10(2):261-77.

13. Abdurrazak M, Rao MU, Ado AB, Mohd KS, Thant Z. Some natural products and their secondary metabolites attributed towards diabetic cure: A review. Int J Pharm Pharm Sci 2015;7:22-8.

14. Rupeshkumar M, Kavitha K, Haldar PK. Role of herbal plants in the diabetes mellitus therapy: An overview. Int J Appl Pharm 2014;6(3):1-3.

15. Sadowska-Bartosz I, Galiniak S, Bartosz G. Kinetics of glycoxidation 
of bovine serum albumin by methylglyoxal and glyoxal and its prevention by various compounds. Molecules 2014;19(4):4880-96.

16. Séro L, Sanguinet L, Blanchard P, Dang BT, Morel S, Richomme P, et al. Tuning a 96-well microtiter plate fluorescence-based assay to identify AGE inhibitors in crude plant extracts. Molecules 2013;18(11):14320-39.

17. Matsuura N, Aradate T, Sasaki C, Kojima H, Ohara M, Hasegawa J, et al. Screening system for the Maillard reaction inhibitor from natural product extracts. J Health Sci 2002;48(6):520-6.

18. Koschinsky T, He CJ, Mitsuhashi T, Bucala R, Liu C, Buenting C, et al. Orally absorbed reactive glycation products (glycotoxins): An environmental risk factor in diabetic nephropathy. Proc Natl Acad Sci U S A 1997;94(12):6474-9.

19. Uribarri J, Peppa M, Cai W, Goldberg T, Lu M, He C, et al. Restriction of dietary glycotoxins reduces excessive advanced glycation end products in renal failure patients. J Am Soc Nephrol 2003;14(3):728-31.

20. Kaushik G, Satya S, Khandelwal RK, Naik S. Commonly consumed Indian plant food materials in the management of diabetes mellitus. Diabetes Metab Syndr Clin Res Rev 2010;4(1):21-40.

21. Dzib-Guerra WD, Escalante-Erosa F, García-Sosa K, Derbré S, Blanchard P, Richomme P, et al. Anti-advanced glycation end-product and free radical scavenging activity of plants from the yucatecan flora. Pharmacognosy Res 2016;8(4):276-80.

22. Pinto MD, Kwon YI, Apostolidis E, Lajolo FM, Genovese MI, Shetty K. Potential of Ginkgo biloba L. leaves in the management of hyperglycemia and hypertension using in vitro models. Bioresour Technol 2009;100(24):6599-609.

23. Yin XF, Jeon YE, Shim JH, Kang IJ. Inhibitory efficacy of Ligularia fischeri against aldose reductase and advanced glycation end products formation. Food Sci Biotechnol 2014;23(6):1747-52.

24. Ramkissoon JS, Mahomoodally MF, Ahmed N, Subratty AH. Relationship between total phenolic content, antioxidant potential, and antiglycation abilities of common culinary herbs and spices. J Med Food 2012;15(12):1116-23.

25. Singh P, Jayaramaiah RH, Agawane SB, Vannuruswamy G, Korwar AM, Anand $\mathrm{A}$, et al. Potential dual role of eugenol in inhibiting advanced glycation end products in diabetes: Proteomic and mechanistic insights. Sci Rep 2016;6:18798.

26. Jariyapamornkoon N, Yibchok-anun S, Adisakwattana S. Inhibition of advanced glycation end products by red grape skin extract and its antioxidant activity. BMC Complement Altern Med 2013;13:171.

27. Yeh WJ, Hsia SM, Lee WH, Wu CH. Polyphenols with antiglycation activity and mechanisms of action: A review of recent findings. J Food Drug Anal 2017;25(1):84-92.

28. Veverka M, Gallovič J, Švajdlenka E, Veverková E, Prónayová N, Miláčková I, et al. Novel quercetin derivatives: Synthesis and screening for anti-oxidant activity and aldose reductase inhibition. Chem Pap 2013;67(1):76-83.

29. Delgado L, Fernandes I, González-Manzano S, de Freitas V, Mateus N, Santos-Buelga C. Anti-proliferative effects of quercetin and catechin metabolites. Food Funct 2014;5(4):797-803.

30. Goodarzi M, Zal F, Malakooti M, Safari M, Sadeghian S. Inhibitory activity of flavonoids on the lens aldose reductase of healthy and diabetic rats. Acta Med Iran 2006;44(1):41-5.

31. Murata M, Irie J, Homma S. Aldose reductase inhibitors from green tea. Food Sci Technol 1994;27(5):401-5.

32. Begum SF, Hemalatha SP. Characterization, in silico and in vitro determination of antidiabetic and anti-inflammatory potential of ethanolic extract of Sargassum wightii. Asian J Pharm Clin Res 2017;10(4):297-301.

33. Coughlan MT, Thorburn DR, Penfold SA, Laskowski A, Harcourt BE, Sourris KC, et al. RAGE-induced cytosolic ROS promote mitochondrial superoxide generation in diabetes. J Am Soc Nephrol 2009;20(4):742-52. 